\title{
Marangoni instability of a heated liquid layer in the presence of a soluble surfactant
}

\section{Oscillatory mode for small Lewis number}

\author{
S. Shklyaev ${ }^{1,2, a}$ and A.A. Nepomnyashchy ${ }^{3, b}$ \\ 1 Department of Chemical Engineering, California Institute of Technology, Pasadena, \\ CA, USA \\ 2 Institute of Continuous Media Mechanics UB RAS, Perm, Russia \\ 3 Department of Mathematics, Technion - Israel Institute of Technology, Haifa, Israel
}

Received 21 January 2011 / Received in final form 25 January 2011

Published online 9 March 2011

\begin{abstract}
We consider the influence of adsorption kinetics on a longwave oscillatory instability in a layer of a binary liquid heated from below. It is shown that an advection of the adsorbed surfactant leads to a strong stabilization of the mode. Qualitative explanation of the numerical results is provided.
\end{abstract}

\section{Introduction}

Longwave monotonic and oscillatory modes of Marangoni convection in a layer of a binary liquid were revealed in Ref. [1]. Later [2], this analysis was extended to the case of small Lewis numbers $(L)$, which takes place for many of binary mixtures including particle suspensions. It was shown, that the monotonic mode remains qualitatively the same as for finite values of the Lewis number. In contrast, for the oscillatory mode smallness of $L$ drastically changes the analysis: novel oscillatory mode emerges. The present paper is devoted to analysis of this mode for a surfactant, when adsorption kinetics becomes important.

\section{Problem formulation}

Let us consider a planar layer of thickness $d$ of a liquid, which contains a soluble surfactant. The binary mixture is placed on a substrate heated from below; a constant gradient of the temperature $-a$ is specified in a liquid at the contact with the substrate. When dependence of the Soret coefficient on the concentration and temperature can be disregarded, the gradient of the bulk (mass) concentration $C$ formed across the layer due to the Soret effect is uniform [3]. The upper free surface is nondeformable, its surface tension linearly depends on both the temperature $T$ and surface

\footnotetext{
a e-mail: shklyaev@psu.ru

b e-mail: nepom@math.technion.ac.il
} 
concentration of the surfactant $\Gamma$. Adsorption kinetics (with $k_{a}$ and $k_{d}$ being the adsorption and desorption rates, respectively) and surface diffusion (with the diffusivity $D_{2}$ ) are taken into account there.

We choose the Cartesian reference frame with $z$-axis normal to the substrate (the $x-y$ plane). Fluid dynamics is governed by the following set of dimensionless equations:

$$
\begin{gathered}
P^{-1}\left(\mathbf{v}_{t}+\mathbf{v} \cdot \nabla \mathbf{v}\right)=-\nabla p+\nabla^{2} \mathbf{v}, \quad \nabla \cdot \mathbf{v}=0, \\
T_{t}+\mathbf{v} \cdot \nabla T=\nabla^{2} T, \quad C_{t}+\mathbf{v} \cdot \nabla C=L \nabla^{2}(C+\chi T),
\end{gathered}
$$

with the boundary conditions

$$
\begin{gathered}
\mathbf{v}=0, \quad T_{z}=-1, \quad C_{z}+\chi T_{z}=0 \quad \text { at } z=0, \\
w=0, T_{z}+B T=0, \quad C_{z}+\chi T_{z}=-K_{a} L^{-1}(C-\Gamma), \\
\mathbf{u}_{z}=-M \nabla_{2}(T+\Gamma), \quad \Gamma_{t}+\nabla_{2} \cdot(\Gamma \mathbf{u})-L_{s} \nabla_{2}^{2} \Gamma=K_{d}(C-\Gamma) \quad \text { at } z=1 .
\end{gathered}
$$

Here $\mathbf{v}=\mathbf{u}+w \mathbf{e}_{z}, \nabla_{2}$ is the two-dimensional projection of the gradient onto the $x-y$ plane, and a subscript denotes partial derivative with respect to a corresponding variable. As the reference values for the length, time, velocity, pressure, temperature, surface and bulk concentration we choose $d, d^{2} / \kappa, \kappa / d, \eta \kappa / d^{2}, a d$, $\sigma_{\Gamma}^{-1} \sigma_{T} a d, k_{d}\left(k_{a} \sigma_{\Gamma}\right)^{-1} \sigma_{T} a d$, respectively, where $\kappa$ is the thermal diffusivity, $\eta$ is the dynamic viscosity, and $\sigma_{T, \Gamma}=-d \sigma / d(T, \Gamma)$.

Boundary-value problem (1)-(4) is governed by the eight dimensionless parameters: $P=\nu / \kappa, B=q d / k_{t h}, M=\sigma_{T} a d^{2} / \eta \kappa, \chi=\alpha_{D} k_{a} \sigma_{\Gamma} / k_{d} \sigma_{T}, L=D / \kappa, L_{s}=$ $D_{2} / \kappa, K_{a}=k_{a} d / \kappa, K_{d}=k_{d} d^{2} / \kappa$. They are the Prandtl, Biot, Marangoni, Soret, Lewis numbers, the surface analogue of the Lewis number, dimensionless adsorption and desorption coefficients, respectively. Here $\nu$ is the kinematic viscosity, $D$ is the bulk diffusivity, $q$ is the heat transfer coefficient according to Newton's law of cooling, $k_{t h}$ is the thermal conductivity, and $\alpha_{D}$ is the Soret coefficient.

In the limit $K_{d} \rightarrow \infty$ we obtain $C(z=1)=\Gamma$, i.e. the problem reduces to that studied in Refs. [1,2]. It is worth noting that the sign of $\chi$ is defined oppositely to that in the cited papers.

Upon substitution of $C-\Gamma$ from the last relation in Eq. (4) into the mass balance condition at $z=1$, one readily obtains:

$$
(C+\chi T)_{z}=-K_{a d} L^{-1}\left[\Gamma_{t}+\nabla_{2} \cdot(\Gamma \mathbf{u})+L_{s} \nabla_{2}^{2} \Gamma\right]
$$

where $K_{a d}=K_{a} / K_{d}=k_{a} /\left(k_{d} d\right)$ is the ratio of the adsorption and desorption coefficients.

Equations (1)-(4) admit an equilibrium base state, corresponding to the conductive distributions of both the temperature and bulk concentration in a motionless liquid:

$$
\mathbf{v}_{0}=0, \quad T_{0}=B^{-1}+1-z, \quad C_{0}=C_{a}+\chi z, \quad \Gamma_{0}=C_{0}(z=1)=C_{a}+\chi .
$$

The reference value of the surface concentration, $\Gamma_{0}$, is the ninth dimensionless parameter governing dynamics of the binary mixture.

Below we consider an oscillatory mode of longwave convection, which takes place for positive $M$ and $\chi$; this ensures the scales for both the bulk and surface concentration to be positive and, in particular, means that $\Gamma_{0}>0$ and $C_{a}>0$. Hence, the following restriction takes place:

$$
\Gamma_{0} \geq \chi
$$


In fact, assuming the Soret coefficient to be constant, we have neglected the factor $C(1-C)$ in the dimensional thermodiffusional flux [3]. This assumption can be justified if $|\chi| / \Gamma_{0}$ is sufficiently small. In further analysis $\Gamma_{0}$ is chosen five times of $\chi$, i.e. the variation of $C_{0}$ due to the Soret effect comprises $20 \%$ of its maximal value.

\section{Linear stability analysis for longwave oscillatory mode}

Linearizing Eqs. (1)-(4) near the base state and introducing two-dimensional normal perturbations of the form $f(x, z, t)=\hat{f}(z) \exp (i k x+\lambda t)$, we obtain

$$
\begin{gathered}
\lambda P^{-1} \hat{u}=-i k \hat{p}+\Delta \hat{u}, \quad \lambda P^{-1} \hat{w}=-\hat{p}^{\prime}+\Delta \hat{w}, \quad i k \hat{u}+\hat{w}^{\prime}=0, \\
\lambda \hat{T}-\hat{w}=\Delta \hat{T}, \lambda \hat{C}+\chi \hat{w}=L \Delta(\hat{C}+\chi \hat{T}), \\
\hat{u}=\hat{w}=\hat{T}^{\prime}=\hat{C}^{\prime}=0 \quad \text { at } z=0, \\
\hat{w}=0, \quad \hat{T}^{\prime}+B \hat{T}=0, \quad \hat{C}^{\prime}+\chi \hat{T}^{\prime}=-K_{a d} L^{-1}\left(\lambda \hat{\Gamma}+\Gamma_{0} i k \hat{u}+L_{s} k^{2} \hat{\Gamma}\right), \\
\hat{u}^{\prime}=-M i k(\hat{T}+\hat{\Gamma}), \quad \lambda \hat{\Gamma}+\Gamma_{0} i k \hat{u}+L_{s} k^{2} \hat{\Gamma}=K_{d}(\hat{C}-\hat{\Gamma}) \quad \text { at } z=1,
\end{gathered}
$$

where the prime denotes the $z$-derivative, $\Delta \equiv d^{2} / d z^{2}-k^{2}$ is the Fourier transform of the Laplace operator, and $\hat{u}$ is the $x$-component of the velocity perturbation.

Hereafter we address the influence of the adsorption kinetics on the longwave oscillatory mode found in Ref. [2] for small values of $L$ (see Case I there). Therefore, we assume both the Lewis numbers and the Biot number to be asymptotically small and rescale the wavenumber and growth rate as follows:

$$
L=\epsilon^{2} l, \quad L_{s}=\epsilon^{2} l_{s}, \quad B=\epsilon^{2} \beta, \quad k=\epsilon K, \quad \lambda=\epsilon^{2} \Lambda .
$$

Note, that unusual scaling $B=O\left(k^{2}\right)$ instead of $B=O\left(k^{4}\right)$ is used [4].

We expand the fields of perturbations and the Marangoni number as follows

$$
\begin{gathered}
\hat{T}=\hat{T}_{0}+\epsilon^{2} \hat{T}_{1}+\ldots, \quad \hat{C}=\hat{C}_{0}+\epsilon^{2} \hat{C}_{1}+\ldots, \quad \hat{p}=\hat{p}_{0}+\epsilon^{2} \hat{p}_{1}+\ldots, \\
\hat{u}=\epsilon\left(\hat{u}_{0}+\epsilon^{2} \hat{u}_{1}+\ldots\right), \quad \hat{w}=\epsilon^{2}\left(\hat{w}_{0}+\epsilon^{2} \hat{w}_{2}+\ldots\right), \quad M=48\left(m_{0}+\epsilon^{2} m_{1}+\ldots\right) .
\end{gathered}
$$

Substituting these expansions into Eqs. (8)-(11), we collect terms of the same order in $\epsilon^{2}$.

The zero-order problem has the following form ( $\hat{C}_{0}$ will be discussed below):

$$
\begin{gathered}
\hat{T}_{0}^{\prime \prime}=0, \quad \hat{p}_{0}^{\prime}=0, \quad \hat{u}_{0}^{\prime \prime}=i K \hat{p}_{0}, \quad i K \hat{u}_{0}+\hat{w}_{0}^{\prime}=0, \\
\hat{u}_{0}=\hat{w}_{0}=\hat{T}_{0}^{\prime}=0 \quad \text { at } z=0, \\
\hat{w}_{0}=\hat{T}_{0}^{\prime}=0, \quad \hat{u}_{0}^{\prime}=-48 i K m_{0} h, \quad \text { at } z=1,
\end{gathered}
$$

where $h \equiv \hat{T}_{0}(z=1)+\hat{\Gamma}_{0}$ is a $z$-independent quantity. This problem has an obvious solution

$$
\begin{aligned}
& \hat{T}_{0}=F, \quad \hat{p}_{0}=-72 m_{0} h, \quad \hat{u}_{0}=-12 m_{0} i K h z(3 z-2), \\
& \hat{w}_{0}=-12 m_{0} K^{2} h z^{2}(z-1) .
\end{aligned}
$$


In the first order we need only the boundary-value problem governing $\hat{T}_{1}$ :

$$
\begin{gathered}
\hat{T}_{1}^{\prime \prime}=\left(K^{2}+\Lambda\right) \hat{T}_{0}-\hat{w}_{0}, \\
\hat{T}_{1}^{\prime}=0 \quad \text { at } z=0, \\
\hat{T}_{1}^{\prime}=-\beta \hat{T}_{0} \quad \text { at } z=1 .
\end{gathered}
$$

The solvability condition for this problem reads

$$
\left(\Lambda+K^{2}+\beta\right) F-m_{0} K^{2} h=0,
$$

this is the first relation coupling the temperature and surface concentration of the surfactant.

In order to obtain the second relation, we appeal to the boundary-value problem governing the perturbation of the bulk concentration:

$$
\begin{gathered}
l \hat{C}_{0}^{\prime \prime}-\Lambda \hat{C}_{0}=\chi \hat{w}_{0}, \\
\hat{C}_{0}^{\prime}=0 \quad \text { at } z=0, \\
\hat{C}_{0}^{\prime}=-K_{a d} l^{-1}\left(\Lambda \hat{C}_{0}+12 m_{0} K^{2} \Gamma_{0} h\right) \quad \text { at } z=1 .
\end{gathered}
$$

Here we have taken into account that in view of the last relation in Eq. (11), $\hat{\Gamma}_{0}=$ $\hat{C}_{0}(z=1)+O\left(\epsilon^{2}\right)$. (This is a consequence of the fact that the adsorption is fast with respect to the diffusion: $K_{a}$ and $K_{d}$ are of order unity, whereas $L \ll 1$.) Solution of this boundary value problem reads:

$$
\hat{C}_{0}=-\frac{12 m_{0} \chi K^{2}}{\Lambda q^{3}}\left[\frac{A \cosh q z-6 \cosh q(1-z)}{\sinh q}-q^{3} z^{2}(z-1)-2 q(3 z+1)\right] h,
$$

with $q^{2}=\Lambda / l$ and $A$ determined by boundary condition (25). The expression $h=$ $F+\hat{\Gamma}_{0}$ provides the second linear equation coupling $F$ and $\Gamma_{0}$ :

$$
h=F-\frac{12 m_{0} \chi K^{2}}{\Lambda} f(q) h, \quad f=q^{-3}\left(A \operatorname{coth} q-6 \sinh ^{-1} q-4 q\right) .
$$

It is clear that $f(q)$ coincides with $\hat{\Gamma}_{0}=\hat{C}_{0}(z=1)$ up to the factor $-12 m_{0} \chi K^{2} / \Lambda$.

The solvability condition for Eqs. (22) and (27) reads:

$$
m_{0} K^{2}=\left(K^{2}+\beta+\Lambda\right)\left[1+12 m_{0} K^{2} \chi \Lambda^{-1} f(q)\right] .
$$

Equation (28) coincides with the corresponding equation in Ref. [2] (cf. Eq. (40) there keeping in mind the inverted signs for $\Lambda$ and $\chi$ ); the only difference is the definition of $f(q)$, which is now influenced by the adsorption kinetics. This complex transcendental equation determines the complex growth rate $\Lambda$. At the marginal stability curve $m_{0}=m_{*}(K)$, the growth rate is pure imaginary $\Lambda=i \Omega$. A parametric representation of $m_{*}(K)$ with $\Omega$ serving as a parameter can be easily obtained from Eq. (28). However, this formula is rather cumbersome, and is not presented here. Note, that the adsorption kinetics is governed by $K_{a d}$ and $\Gamma_{0}$ only. Below we set $l=1$, which is equivalent to choosing $\epsilon=\sqrt{L}$. 


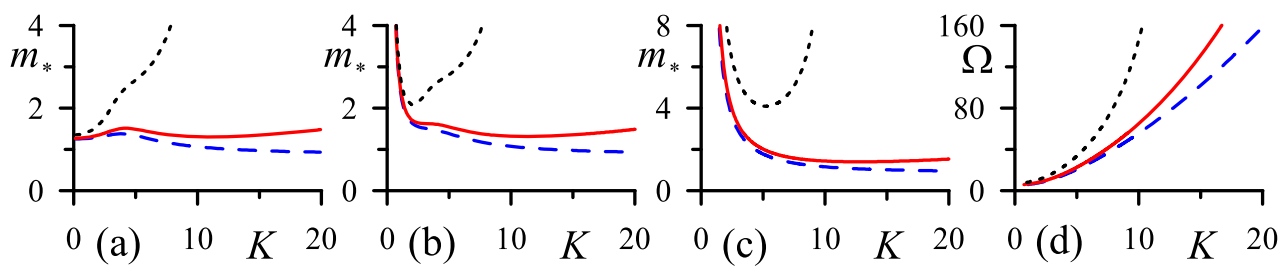

Fig. 1. (Color online) Results of computations for $\Gamma_{0}=1, \chi=0.2$. Panels (a), (b), and (c) - marginal stability curves $m_{*}(K)$ for $\beta=0,1,10$, respectively. Domains of stability are below the curves. Panel (d) - variation of the frequency $\Omega$ of neutral perturbations on $K$ for $\beta=10$. For smaller $\beta$ the variation of $\Omega$ with $K$ is similar. Dashed (blue), solid (red), and dotted (black) lines correspond to $K_{a d}=0,0.001$, and 0.005 , respectively.

\section{Results of calculations}

Recall [2], that for $K_{a d}=0$ a typical neutral stability curve consists of two parts (see dashed lines in Fig. 1): One of them, belonging to smaller $K$, is intimately related to the longwave oscillatory mode found in Ref. [1]. Another one materializes a global minimum at $K \approx 100$. The latter part is qualitatively similar to the neutral stability curve for the shortwave mode (with finite $k$ ) found numerically at rather small, but finite $L$. With increase in $\beta, m_{*}$ grows fast for smaller $K$, as shown in Fig. 1(b), which results in disappearing the corresponding minimum (Fig. 1(c)); for larger $K m_{*}(K)$ changes slightly.

An increase in $K_{a d}$ leads to strong damping of perturbations, which is especially pronounced for larger $K$, see Fig. 1 . Moreover, at $K \gg 1$ the neutral stability curve completely disappears: at $K=K_{*}$ the neutral stability curve has a vertical asymptote and for $K>K_{*}$ there is no instability at all. This very value $K_{*}$ decreases fast as $K_{a d}$ grows.

Results of minimization of $m_{*}(K)$ are presented in Fig. 2. It is clear that with increase in $K_{a d}$, there appears a competition between the two modes belonging to the minima of $m_{*}(K)$ at larger and smaller $K$. At small enough $K_{a d}$ the mode with larger $K$ remains critical, but at $K_{a d}>K_{a d}^{(c)}$ the global minimum takes place at smaller $K$. Here $K_{a d}^{(c)}$ is determined by the intersection of the corresponding lines in Fig. 2(a); both the critical values of $K$ and $\Omega$ have jumps at this value of $K_{a d}$, see Fig. 2(b) and Fig. 2(c), respectively.

For $\beta=0$, the minimum at smaller $K$ materializes the true longwave asymptotics with $K_{c}=0$ and

$$
m_{c}=\frac{1+K_{a d}}{1-\chi+K_{a d}\left(1-12 \Gamma_{0}\right)} ;
$$

the frequency is proportional to $K^{2}$ for small $K$. Equation (29) is a natural extension of results of Ref. [1] to the impact of the adsorption kinetics.

At larger values of $\beta$, when the neutral curve is unimodal (Fig. 1(c)), $K_{c}$ diminishes fast with growth of $K_{a d}$, which is accompanied by a fast increase in $m_{c}$ and decrease in $\Omega_{c}$, see dashed lines in Fig. 2. Thus, even in the absence of competition of two minima, the evolution of critical parameters with $K_{a d}$ remains qualitatively similar.

\section{Discussion}

Let us briefly discuss the mechanism of stabilization found numerically, dealing with the case of large $K$ and, hence, large $\Omega$ and $|q|$ (see Fig. 1(d)). Mathematically this 

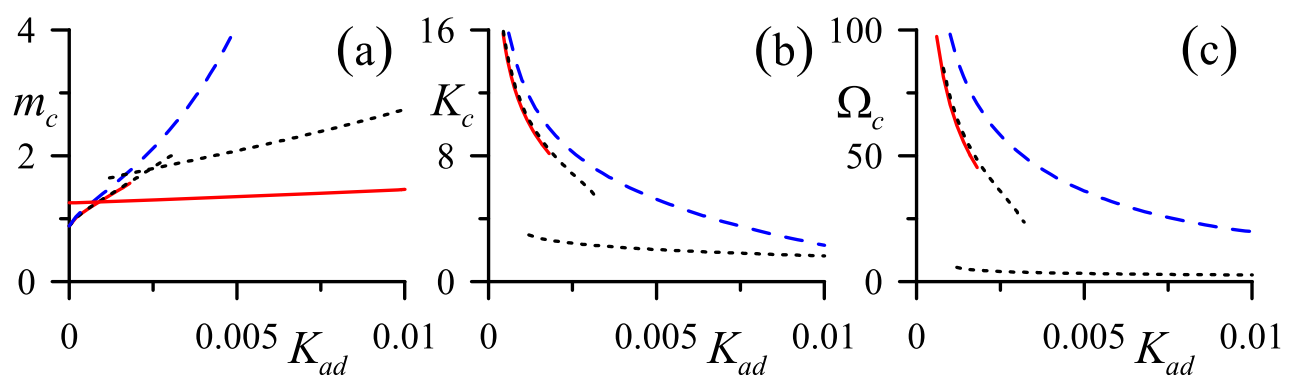

Fig. 2. (Color online) Variation of the critical Marangoni number $m_{c}$ (a), wavenumber $K_{c}$ (b), and frequency $\Omega_{c}$ with $K_{a d}$ for $\chi=0.2 ; \Gamma_{0}=1$. Solid (red), dotted (black), and dashed (blue) lines correspond to $\beta=0,1,10$, respectively. Almost horizontal solid line in panel (a) corresponds to the longwave instability $\left(K_{c}=\Omega_{c}=0\right)$ for $\beta=0$, Eq. (29).
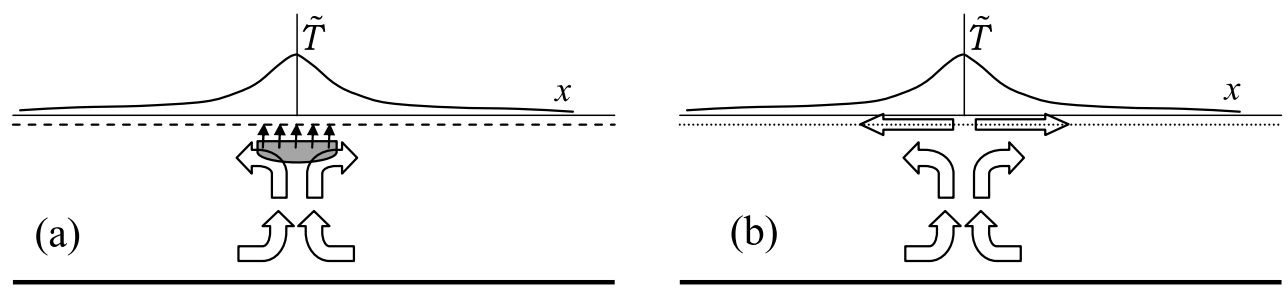

Fig. 3. Sketch of the evolution of the initial fluctuation in form of a hot spot. (a) $-K_{a d}=0$, the lack in the concentration (grey domain) penetrates at the free surface due to diffusion. (b) $-K_{a d} \neq 0$, the initial "Pearson" flow diminishes the surface concentration $\Gamma$ near the spot.

stabilization stems from the change of the type of boundary condition for $\hat{C}_{0}$ at $z=1$. Indeed, for $K_{a d}=0$, Eq. (25) reduces to the second-type boundary condition and the perturbation of the concentration at the free surface is small: $f(q)=O\left(q^{-1}\right)$. (Recall, that $f(q)$ differs from $\hat{C}_{0}(z=1)$ only by the constant factor.) As shown in Ref. [2], $\Omega \sim K^{8 / 5}$ at large $K$, i.e. a subtle balance of the mass transfer takes place for this instability mode: whereas $\hat{C}_{0}$ in the bulk is large in comparison with the disturbance of the temperature $\hat{C}_{0}(z) / F=O\left(K^{2 / 5}\right)$, at the free surface one has the opposite inequality: $F / \hat{C}_{0}(z=1)=O\left(K^{2 / 5}\right)$.

In contrast, for any $K_{a d} \neq 0$ the boundary condition is of the third type and the perturbation of the concentration is finite: $f(q)=O(1)$. This destroys the abovementioned balance of the surfactant flux and drastically changes the analysis carried out in Ref. [2] for large $K$. It is clear, that for any finite $K_{a d}$ right-hand part of Eq. (25), containing large factors $K^{2}$ and $\Lambda$ cannot be neglected in comparison with the left-hand part.

The mechanism of the oscillatory mode at $K_{a d}=0$ and $K \gg 1$ can be explained as follows. For large $|q|$ a diffusive boundary layer develops near the free surface according to Eq. (26): the sharp change of the $\hat{C}_{0}$ occurs there. Let us consider nonlinear evolution of a fluctuation in the form of a hot spot at the free surface. The spot generates a convective flow of Pearson's type [5], with the fluid raising to the spot and the stagnation point at the free surface, see Fig. 3(a). Since the stability threshold is close to $m_{*}=1$ obtained by Pearson, monotonic Pearson's mode, coupling the disturbances of the velocity and temperature, decays slowly. At 
this stage, the surfactant behaves as a passive scalar and its value becomes smaller near the stagnation point at the free surface (see the grey area in Fig. 3(a)). Since the normal component of the velocity vanishes at the free surface, the concentration disturbance is small there as it has been stated before. To emphasize this fact, we plot the free surface to be untouched by the grey area. Then the lack of the surfactant slowly diffuses onto the free surface as shown by arrows and triggers an intensive solutocapillary motion of the opposite direction, which sweeps fast the inhomogeneity in concentration. Indeed, the "solutal" Marangoni number, $M_{s}=-M \chi / L$ is negative (stable) and its absolute value is large. After the fast decay of the flow, again, slowly decaying Pearson's mode dominates and the process is repeated.

In presence of the adsorption kinetics and advection of the surface phase the situation is qualitatively different. Pearson's flow immediately transports the surface phase of the surfactant from the stagnation point, see Fig. 3(b), which restores the initial value of the surface tension and, hence, hinders the onset of Pearson's convective flow.

\section{Conclusions}

We study the influence of the adsorption kinetics on the longwave oscillatory mode found for the binary fluids with extremely small Lewis numbers in Ref. [2]. It is shown, that advection of the surface phase of the surfactant leads to a strong stabilization of the oscillatory mode and, in turn, conventional longwave oscillatory mode [1] becomes critical in a wide interval of the Soret number.

Authors acknowledge the support of Israel Science Foundation (grant 812/06), joint grants of the Israel Ministry of Sciences (Grant No. 3-5799) and the Russian Foundation for Basic Research (Grant No.09-01-92472). The work is also supported by the European Union via FP7 Marie Curie scheme Grant PITN-GA-2008-214919 (MULTIFLOW).

\section{References}

1. A. Oron, A.A. Nepomnyashchy, Phys. Rev. E 69, 016313 (2004)

2. S. Shklyaev, A.A. Nepomnyashchy, A. Oron, Phys. Fluids 21, 054101 (2009)

3. S. Slavtchev, G. Simeonov, S. Van Vaerenbergh, J.C. Legros, Int. J. Heat Mass Transfer 42, 3007 (1999)

4. A. Podolny, A. Oron, A.A. Nepomnyashchy, Phys. Fluids 17, 104104 (2005)

5. J.R.A. Pearson, J. Fluid Mech. 4, 489 (1958) 